\title{
Analysis of the automatic transmission fault diagnosis based on Bayesian network sensitivity
}

\author{
Luo Jin", Ma Qihua, Luo Yiping \\ Faculty of Automotive Engineering, Shanghai University of Engineering and Science, Shanghai, China \\ Email address: \\ 84276661@qq.com (Luo Jin), mqh0386@163.com (Ma Qihua), lyp777@sina.com (Luo Yiping)
}

\section{To cite this article:}

Luo Jin, Ma Qihua, Luo Yiping. Analysis of the Automatic Transmission Fault Diagnosis Based on Bayesian Network Sensitivity. American Journal of Networks and Communications. Vol. 3, No. 2, 2014, pp. 25-28. doi: 10.11648/j.ajnc.20140302.12

\begin{abstract}
Automobile automatic transmission is a complex system integrates machine, electricity, liquid, in terms of its fault diagnosis has the characteristics of uncertainty and high complex correlation, thus put forward the Bayesian network based on the theory of probability and theory of the fault tree for fault diagnosis methods of automobile automatic transmission, it has a certain value for application. Take a failure automatic transmission as an example, analyzes the causes of the automatic transmission fault and the relationship between the affecting factors, according to the properties of the automatic transmission fault establishes a Bayesian network model, and through calculating sensitivity to determine the probability of a certain causes to the automatic transmission fault. The results show that the method is operable in automatic transmission fault diagnosis, diagnosis results are accurate and credible.
\end{abstract}

Keywords: Automatic Transmission, Bayesian Network, Sensitivity

\section{Introduction}

Automatic transmission with a lot of advantage, such as good transmission ratio, improve the comfort and trafficability of the vehicle, reduce driving operation fatigue strength and reduce the emissions, is widely used in modern cars. At the same time, the automatic transmission is the complex machinery and equipment with a collection of mechanical, electronic control, and hydraulic transmission, its fault identification and diagnosis is difficult because the complexity of its structure and working principle. Therefore, it's have great significance to establish an operable, accurate and rapid diagnostic methods to ensure transmission can work reliably.

Since the automatic transmission was born to now, automatic transmission fault diagnosis technology has been an important part of automatic transmission technology research and development. At present, foreigner studying an online automatic transmission fault diagnosis system that diagnosis range is more broader, its principle is based on the entire model of the automatic transmission, make full use of existing sensor information of automatic transmission, as much as possible to obtain meaningful information for faults. Jin-Oh Hahn ${ }^{[1]}$ proposed a algorithm based on state observer to evaluate the oil pressure of the shift actuator, it's have great significance for the improvement of the automatic transmission control technology and study of the online fault diagnosis system. In domestic, $\mathrm{Hu} \mathrm{Ning}^{[2]}$ et al proposed function analysis methods of the automatic transmission fault, according to the function of the of each components, to study the component failure impact on the automatic transmission. Qin Guihe ${ }^{[3]}$ proposed that use exists in measurement data and local power transmission system model and redundant information between the structure logical relationship to detect and diagnose the faults of the sensors, actuators and AMT parts. Wei Shaoyuan ${ }^{[4]}$ proposed to diagnose automotive automatic transmission fault based on fuzzy theory and put forward to set up a system of automatic transmission fault diagnosis. Overall, the current domestic research on fault diagnosis of the automatic transmission also stay on how to solve some targeted specific example.

This paper make a detailed analysis of the automatic transmission fault diagnosis, apply for Bayesian network in the field of automatic transmission failure, and by calculating the sensitivity with examples to verify the feasibility of Bayesian networks for automatic transmission failure. On the basis of Bayesian networks, establish a network diagram of the automatic transmission fault, According to the specific fault reasoning procedure and sensitivity calculation to achieve automatic transmission fault diagnosis, to solve the uncertain reasoning problems in 
the process of found fault reason. A comprehensive of preliminary testing, failure analysis, probability calculation and fault recognition, thus forming a complete automatic transmission fault diagnosis system based on Bayesian network. The results show that Bayesian network have realized the optimization of judgment steps, it has good effect.

\section{Introduction to Bayesian Networks}

Bayesian networks is a probabilistic network, which is based on probabilistic reasoning graphical network, and the basis of this network is Bayesian probability formula. Bayesian network use directed graph to describe the relationship between probability theory, it is suitable for the uncertainty and probabilistic things, applied to related issues what conditionally dependent on a variety of control factors, is a kind of probability inference technology, can use probability theory to deal with the uncertainty produced by the knowledge relationship because of conditions correlation.

In simple terms, the Bayesian network diagram is a kind of cycle directed graph and related parameters properties, it's consist of the model structure and related parameters. In Bayesian network, the node represent the variable, the contact between them represented by directed arc, usually indicates the cause node to result node. So that can draw a causal relationship between each node and to describe the degree of variable may affect another variable by probability. In probabilistic reasoning, the random variable is used to represent events or things, through the random variable instance into various instances, it can be modeling a series of events or the existing state of things. Based on the theory of Bayesian probability, it can be calculated the joint probability under the a certain condition. Fig. 1 is a simple but typical example of Bayesian network (omitting the conditional probability values).

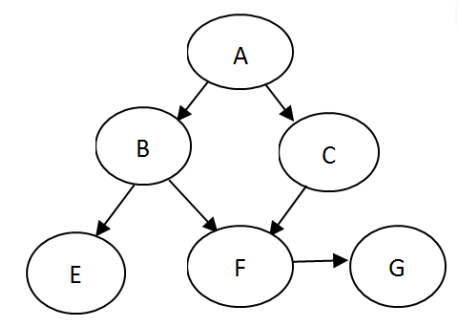

Fig 1. Sample of Bayesian network structure

Assuming that there are $\mathrm{n}$ variables, to remember $X_{1}, X_{2}, \cdots, X_{n}$, to contain a combination of variable distribution $P\left(X_{1}, X_{2}, \cdots, X_{n}\right)$, then decomposed this joint distribution with the chain rule, see equation (1) :

$$
P\left(X_{1}, X_{2}, \cdots, X_{n}\right)=\prod_{i=1}^{n} P\left(X_{i} \mid X_{1}, X_{2}, \cdots, X_{i-1}\right)
$$

Assuming the existence of $\pi\left(X_{i}\right) \in\left\{X_{1}, X_{2}, \cdots, X_{i-1}\right\}$ an $\operatorname{arbitrary} X_{i}$, and to set the conditions under $\pi\left(X_{i}\right), X_{i}$ and $\left\{X_{1}, X_{2}, \cdots, X_{n}\right\}$ in the other variables are independent of each other, then formula (1) can be rewritten as formula (2) :

$$
P\left(X_{1}, X_{2}, \cdots, X_{n}\right)=\prod_{i=1}^{n} P\left(X_{i} \mid \pi\left(X_{i}\right)\right)
$$

$A$ and $B$ are provided as two random variables, the probability of $A$ occurrence is $P(A), \quad(P(A)>0)$, the probability of $B$ occurrence is $P(B),(P(B)>0)$, the probability of $B$ occurrence conditions for $A$ occurrence is $P(B \mid A)$, there is a multiplication formula, see equation (3) :

$$
P(A B)=P(B \mid A) P(A)
$$

Let $A_{1}, A_{2}, \cdots, A_{n}$ constitute an integral event, and have $P\left(A_{i}\right)>0$, the total probability of the event $B$ has a formula, see equation (4) :

$$
P(B)=\sum_{i=1}^{n} P\left(A_{i}\right) P\left(B \mid A_{i}\right)
$$

Equation (2) (4) can be calculated the joint distribution function between the variables of each factor in the accident.

\section{The Construction of a Bayesian Network Diagram Automatic Transmission Fault}

Different manufacturers and different car manufacturers with different types of automatic transmission. Though the structure of different types of automatic transmission are different, the basic function and working principle of various components are basically the same. In general, the automatic transmission is consist of external operating system, hydraulic transmission device, mechanical structure, hydraulic control system and circuit control system.

Automatic transmission fault caused by many reasons, but the occurrence probability of reasons is different. Need to find a greater probability caused by fault reasons from the symptoms, therefore, put the automatic transmission fault classification is very important. Automatic transmission fault generally have the following symptoms, respectively: no forward gears and reverse gears, big shift impact, shift up too late, can't shift gears, hang power engine shut down immediately, automatic transmission oil temperature is too high, appearing vibration in the process of driving, the transmission gear jumping, and so on. Removing some of the new cars because its quality problems causes the fault of the automatic transmission, but with the decline in the status of automatic transmission technology and life shortening, 
there will be produce a series of fault, if the fault not resolved quickly often cause serious damage, however the common failure usually manifested through certain phenomenon, so it's important as soon as possible to find out the fault reason through the phenomenon. Assuming that automatic transmission malfunction is the parent node, its associated child nodes respectively hydraulic torque converter, mechanical drive mechanism, pressure of automatic transmission oil, pressure of the governor, pressure of the throttle, circuit control system, locking clutch, and so on. The above factors listed in sequence into Bayesian network diagram (see fig. 2). Analysis is as follows: (1) influence most is the oil, followed is the circuit, then the mechanical fault and hydraulic torque converter fault; (2) the focus of fault diagnosis is the oil and circuit.

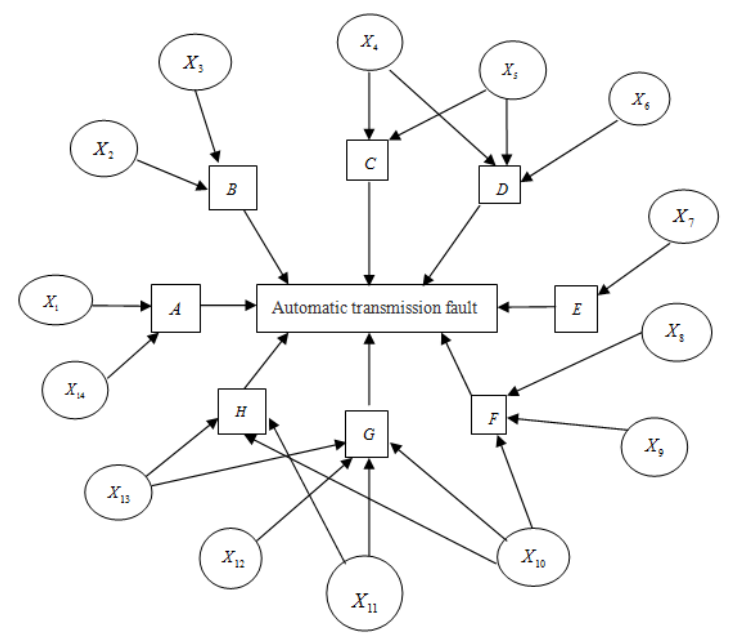

Fig 2. Bayesian network diagram of automatic transmission fault

A: no forward gears and reverse gears; B: big shift impact; C: can't shift gears; D: shift up too late; E: hang power engine shut down immediately; F: automatic transmission oil temperature is too high; G: appearing vibration in the process of driving; $\mathrm{H}$ : the transmission gear jumping; $X_{1}$ : automatic transmission light-leaking; $X_{2}$ : Energy storage shock absorber of failure; $X_{3}$ : Work oil pressure is high; $X_{4}$ : throttle pressure is high; $X_{5}$ : the governor oil pressure too low; $X_{6}$ : circuit control system failure; $X_{7}$ : hydraulic torque converter failure; $X_{8}$ : cooler pipeline or blockage in a one-way valve; $X_{9}$ : the oil sump level is too low; $X_{10}$ : clutch skid; $X_{11}$ : shift institution failure; $X_{12}$ : engine failure; $X_{13}$ : electronic components failure; $X_{14}$ : the main serious leaks oil

The figure 2 is a directed acyclic graph, actually it's a Bayesian network, the fault diagnostic process can be seen as a probabilistic Bayesian network inference process, and thus can be use Bayesian network as the representation and solving methods of the uncertain problems in fault diagnosis process, so based on the Bayesian network graph can also understand that: solving object is automatic transmission fault, the fault produced by the oil circuit, circuit, mechanical fault, fault of hydraulic torque converter; its failure of automatic transmission phenomenon is bad working conditions of oil, circuit control system, governor, locking clutch, hydraulic torque converter.

Using Bayesian network for automatic transmission failure analysis in accordance with people's normal thinking. When the fault happens, firstly, you will be to determine the fault symptoms, and then judge the character of failure and types, according to the information to choose the most suitable solution finally. By Bayesian network probabilistic inference, the fault will be sorted according to the probability of risk events, determine the priority for troubleshooting.

In a word, the Bayesian figure can be described as full of information, it can play a big role in the automatic transmission fault diagnosis.

\section{The calculation of Sensitivity Analysis Method Based on Bayesian Network}

\subsection{The Definition of Parameter Sensitivity Analysis}

To introduce the method of sensitivity analysis for the fault diagnosis of complex systems, the priority task is to determine the related issues of parameter sensitivity and node sensitivity, let's introduce relevant definitions below.

Definition 1 Parametric sensitivity: In a Bayesian network, assuming that there is a parameter $\theta=P\left(b_{i} \mid \pi\right)$ on node $B$, the value of target node $A$ is $a$, value of evidence is $e$, then the sensitivity of parameters of $\theta$ can be expressed as follows:

$$
I(\theta)=\frac{1}{r s} \sum_{a, e} \frac{\partial P(a \mid e)(\theta)}{\partial \theta}
$$

Where $r$ and $s$ represent the number of values of $A$ and $e$.

Definition 2 Node sensitivity: Suppose there are two node $B$ and node $A$ in a Bayesian network and there are edges from $B$ to $A$, the parameter $\theta=P\left(b_{i} \mid \pi\right)$ for node $B$, the value of target node $A$ is $a$, value of evidence is $e$, then the sensitivity of node $B$ with respect to the node $A$ can be expressed as follows:

$$
E I(B \rightarrow A)=\frac{1}{r t} \sum_{j=1}^{r} \sum_{i=1}^{t} I\left(\theta_{i j}\right)
$$

Where $r$ and $t$ represent the number of values of $A$ and $B$. A more important node, we call it is a high sensitivity node.

Due to the particularity of fault diagnosis, we can simplify the following formula (5):

$$
I(\theta)=\frac{\partial P(A=a)(\theta)}{\partial \theta}
$$

The corresponding formula (6) simplified as follows: 


$$
E I(B \rightarrow A)=\frac{1}{t} \sum_{i=1}^{t} I\left(\theta_{i}\right)
$$

By the simplify formula (8), we can see, the higher sensitivity of the parent node, the bigger influence of state and state parameters to child nodes stay normal working, therefore, when the parent node produce problems, it is easy to cause the abnormal state of relatively child nodes.

\subsection{Process of SA_FD Algorithm ${ }^{[5]}$}

As shown in Figure 2, when a child node have multiple parent nodes, according to the formula (8), we can determine which node should bear the greatest responsibility for the child node produce fault, thus in the process of fault diagnosis system, avoid finding fault blindly, improve the efficiency of fault diagnosis.

Put forward a kind of fault diagnosis algorithm combining with Bayesian network sensitivity analysis: SA_FD (sensitivity analysis fault diagnosis), the whole process of diagnosis algorithm is summarized as follows:

Step 1 Abnormal events occur through the system, the system state of the parent node;

Step 2 Suppose through step1 to find the parent node are $X_{1}, X_{2}, \cdots, X_{n}$, then according to its sensitivity size detect abnormal node;

Step 3 Judge the abnormal nodes if it have parents, if it have then carried out step 4 , if the abnormal modes don't have parent node, so the faulty node is the abnormal nodes;

Step 4 Repeat step 3 until find out the fault node.

\section{Examples of Application}

Figure 2 is about Bayesian network diagram of automatic transmission malfunction, select one of the $G$ child node as the research object, the network parameters see literature ${ }^{[6]}$, when computing child node have more than one parent node appear failure, the size of its parent node CPT (Conditional Probability Distribution) can be obtained according to variant $P\left(X_{i} \mid H\right)=\frac{P\left(H X_{i}\right)}{P(H)}$ of the formula(3), here only show CPT change size of each parent node, the calculation results as shown in tab. 1.

Tab 1. Change of the parent node CPT

\begin{tabular}{lll}
\hline Child node & Parent node & Change of the CPT \\
\hline$G$ & $X_{10} 、 X_{11} 、 X_{12} 、 X_{13}$ & $X_{10}>X_{12}>X_{11}>X_{13}$ \\
\hline
\end{tabular}

SA_FD algorithm is not only need to consider the change degree of parent node state, also need to consider the influence degree of parent node's state parameter for the state of "normal" child nodes, the influence degree is obtained through sensitivity function. According to the formula (8), we can calculate the sensitivity of each node relative to the its child nodes. The calculation results are shown in tab. 2.
Tab 2. Sensitivity of nodes

\begin{tabular}{lcccccccc}
\hline $\begin{array}{l}\text { Child } \\
\text { node }\end{array}$ & \multicolumn{2}{c}{ Parent node } & \multicolumn{5}{c}{ Sensitivity } \\
\hline$G$ & $X_{10}$ & $X_{11}$ & $X_{12}$ & $X_{13}$ & 0.79 & 0.32 & 0.41 & 0.28 \\
\hline
\end{tabular}

Derived from Table 2, when there is too much vibration in the process of driving, the probability is bigger caused by the $X_{10}$ "clutch skid".

For other associated nodes, can be treated using similar methods, respectively obtain the sensitivity of a certain parent node to its child nodes, thus gain the conclusion that this child node (fault symptoms) occurs when the occur probability of a certain parent node (fault reason) is bigger.

\section{Conclusion}

In this paper, sensitivity analysis based on Bayesian networks is applied to fault diagnosis in the field of automotive automatic transmission, when a fault occurs, quantitatively calculated the sensitivity between the child nodes and the parent nodes, thus obtained a conclusion that the bigger probability what a certain cause lead to the automatic transmission fault, improve the accuracy of diagnostic results, saving a lot of time and effort at same time. However, the study objects are limited to static Bayesian network model in this paper, remains to be further put forward a more efficient and precise fault diagnosis method for the dynamic Bayesian network model.

\section{References}

[1] Jin-Oh Hahn, Jae-Woong Hur, Young Man Cho and Kyo Lee. Robust Observer-Based Monitoring of A Hydraulic Actuator In A Vehicle Power Transmission Control System. Proc. Of the 40th IEEE Conference on Decision and Control (pp. 522-528)

[2] Hu Ning, Hu Jia, The function of the automatic transmission failure analysis. The technology of automotive, 2003(8):39-41.

[3] Qin Guihe, Ge Anlin, Lei Yulong. On-line fault diagnosis system of automated mechanical transmission. Journal of agricultural mechanization, 2001(32)5:75-77.

[4] Wei Shaoyuan, Wang Dongmei, Zhang Zhongyang. Automotive automatic transmission based on the theory of fuzzy fault diagnosis system. Mechanical design and manufacturing, 2011 (1):230-232.

[5] Yuan Zheng. Bayesian network sensitivity analysis method and its application in complex system fault diagnosis. [Master's degree thesis]. Hefei university of technology,2012.

[6] Liu Xuejun, Liu Cunxiang, Zhang Erli. Bayesnets application in vehicle gearbox fault analysis. Journal of Nanning College for Vocational Technology. 2013(18) 6:90-92. 